J. Gynäkol. Endokrinol. CH 2021 · 24:147-149 https://doi.org/10.1007/s41975-021-00209-9 Angenommen: 13. August 2021 Online publiziert: 27. August 2021

๑) Der/die Autor(en) 2021
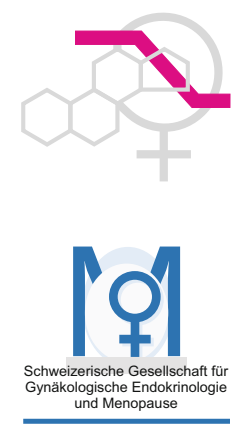

\section{Originalpublikation}

Dotlic J, Radovanovic S, Rancic B, Milosevic B, Nicevic S, Kurtagic I, Markovic N, Gazibara T (2021) Mental health aspect of quality of life in the menopausal transition. J Psychosom Obstet Gynaecol. Mar;42(1):40-49. https://doi. org/10.1080/0167482X.2020.1734789

\section{Hintergrund}

Es gibt viele Hinweise dafür, dass die Wechseljahre mit einem erhöhten Risiko für eine mentale Gesundheitsbeeinträchtigung verbunden sind [1,2]. Das Ziel der aktuellen Studie war es, Faktoren, die mit einer reduzierten mentalen Lebensqualität assoziiert sind, zu identifizieren.

\section{Zusammenfassung}

Die transversale Observationsstudie umfasst 500 Frauen im Alter von 40 bis 65 Jahren, von denen $53,4 \%$ prä-/ peri- und $46,6 \%$ postmenopausal waren. Neben soziodemografischen Daten wurde mithilfe der validierten Fragebögen Women's Health Questionnaire (WHQ; Domänen Depression, Angst,

Nachdruck mit freundlicher Genehmigung aus dem Newsletter der Deutschen Menopause Gesellschaft e.V. und der Schweizerischen Gesellschaft für Gynäkologische Endokrinologie und Menopause.

\title{
Petra Stute
}

Abteilung für Gynäkologische Endokrinologie und Reproduktionsmedizin, Universitätsklinik für Frauenheilkunde, Inselspital Bern, Bern, Schweiz

\section{Mentale Aspekte der Lebensqualität während der Wechseljahre}

Vergesslichkeit, Insomnie) und Kurzform des SF-36 (mentaler Subscore) die mentale Lebensqualität erfasst. Bezugszeitraum war der zurückliegende Monat. Mithilfe eines adjustierten, linearen Regressionsmodells wurden die mit einer schlechteren mentalen Lebensqualität assoziierten Faktoren berechnet. Frauen mit psychischer oder maligner Erkrankung wurden ausgeschlossen. Nur wenige Frauen wandten zum Untersuchungszeitpunkt eine Hormonersatztherapie (HRT) an (Postmenopause 4,3\%, Prä-/Perimenopause 7,5\%).

Insgesamt war die mittlere mentale Gesundheit bzw. Lebensqualität recht gut. Depression, Vergesslichkeit und Insomnie zeigten keinen Gruppenunterschied, nur Angststörungen traten bei prä-/perimenopausalen Frauen signifikant seltener als bei postmenopausalen Frauen auf.

Für prä-/perimenopausale Frauen wurden folgende Assoziationen errechnet: 1) Ein niedrigeres Einkommen $(p=0,046)$ war mir einem höheren Depressionslevel verbunden, 2) ein höherer Body-Mass-Index (BMI; $p=0,045)$ war mit einem höheren Angstlevel verbunden, 3) eine gynäkologische Komorbidität (z. B. Ovarialzyste, Uterus myomatosus, Uterusprolaps; $p=0,017)$ sowie menopausale Symptome $(p=0,039)$ waren mit mehr Vergesslichkeit verbunden,
4) Zusammenleben mit einem Partner ( $p=0,036)$, Alkoholkonsum $(p=0,036)$ und mehr Bewegung $(p=0,041)$ waren mit einem besseren Schlaf verbunden.

Für postmenopausale Frauen wurden folgende Assoziationen errechnet: 1) Das Risiko für eine Depression war bei Frauen, die am Stadtrand wohnten $(p=0,009)$, unverheiratet waren $(p=0,038)$ und einer sitzenden Tätigkeit nachgingen $(p=0,049)$, höher, 2) ein niedriger Bildungsgrad $(p=0,030)$ war mit einem höheren Angstlevel verbunden, 3) ein niedriger Bildungsgrad $(p=0,045)$ sowie menopausale Symptome $(p=0,044)$ waren mit einem schlechteren Gedächtnis assoziiert, 4) fehlende regelmässige Freizeitaktivitäten $(p=0,031)$ waren mit einem besseren Schlaf verbunden. Die Autoren empfehlen, dass Frauen in den Wechseljahren nicht nur bzgl. des Managements von menopausalen Symptomen beraten werden sollten, sondern auch hinsichtlich individueller Faktoren und ihres Verhaltens.

\section{Kommentar}

Neben menopausalen Symptomen beeinflussen individuelle, soziale und Verhaltensfaktoren die mentale Lebensqualität von Frauen in den Wechseljahren. Das ist nicht überraschend. Interessant ist, dass menopausale Symptome sich negativ 
auf das Gedächtnis, nicht aber auf den Affekt und Schlaf auswirkten. Allerdings wurde das Ausmass von menopausalen Symptomen (Frequenz, Intensität) nicht erfasst. Unverständlich (auch für die Autoren) bleibt, warum regelmässige Freizeitaktivitäten sich in Abhängigkeit vom Menopausenstatus günstig bzw. ungünstig auf den Schlaf auswirken sollen (73\% in beiden Gruppen gaben regelmässige Freizeitaktivitäten an). Möglicherweise gab es Überschneidungen in den Items „Freizeitaktivität“ und „körperliche Aktivität"!? Da postmenopausale Frauen einen signifikant höheren BMI hatten und signifikant häufiger an chronischen Erkrankungen als prä-/perimenopausale Frauen litten, könnte vermehrte körperliche Aktivität in der Freizeit evtl. mit den Schlaf kompromittierenden Schmerzen verbunden sein. Die Empfehlung der Autoren ist zu unterstützen, wobei v.a. der Aspekt der Partizipation hervorgehoben werden sollte. Möglicherweise hilft auch hier eine HRT, die in einer älteren prospektiven Kohortenstudie in Deutschland die günstige psychoneuronale Systemwirkung der HRT sowohl auf kognitiver, psychomotorisch-reaktiver, emotional-sozialer und psychovegetativer Ebene beschrieben hat [3].

\begin{tabular}{|c|c|}
\hline \multicolumn{2}{|c|}{ Korrespondenzadresse } \\
\hline & $\begin{array}{l}\text { Prof. Dr. Petra Stute } \\
\text { Abteilung für Gynäkologische } \\
\text { Endokrinologie und } \\
\text { Reproduktionsmedizin, } \\
\text { Universitätsklinik für } \\
\text { Frauenheilkunde, Inselspital } \\
\text { Bern } \\
\text { Friedbühlstrasse 19, } \\
3010 \text { Bern, Schweiz } \\
\text { petra.stute@insel.ch }\end{array}$ \\
\hline
\end{tabular}

Funding. Open access funding provided by University of Bern

Interessenkonflikt. P. Stute gibt an, dass kein Interessenkonflikt besteht.

Open Access. Dieser Artikel wird unter der Creative Commons Namensnennung 4.0 International Lizenz veröffentlicht, welche die Nutzung, Vervielfältigung, Bearbeitung, Verbreitung und Wiedergabe in jeglichem Medium und Format erlaubt, sofern Sie den/die ursprünglichen Autor(en) und die Quelle ordnungsgemäß nennen, einen Link zur Creative Commons Lizenz beifügen und angeben, ob Änderungen vorgenommen wurden.
Die in diesem Artikel enthaltenen Bilder und sonstiges Drittmaterial unterliegen ebenfalls der genannten Creative Commons Lizenz, sofern sich aus der Abbildungslegende nichts anderes ergibt. Sofern das betreffende Material nicht unter der genannten Creative Commons Lizenz steht und die betreffende Handlung nicht nach gesetzlichen Vorschriften erlaubt ist, ist für die oben aufgeführten Weiterverwendungen des Materials die Einwilligung des jeweiligen Rechteinhabers einzuholen.

Weitere Details zur Lizenz entnehmen Sie bitte der Lizenzinformation auf http://creativecommons.org/ licenses/by/4.0/deed.de.

\section{Literatur}

1. de Kruif M, Spijker AT, Molendijk ML (2016) Depression during the perimenopause: a metaanalysis. J Affect Disord 206:174-180. https://doi. org/10.1016/j.jad.2016.07.040

2. Hu LY, Shen CC, Hung JH, Chen PM, Wen $\mathrm{CH}_{\text {, }}$ Chiang YY, Lu T (2016) Risk of psychiatric disorders following symptomatic menopausal transition: a nationwide population-based retrospective cohort study. Medicine (Baltimore) 95(6):e2800. https://doi.org/10.1097/MD.0000000000002800

3. Meissner-Pöthig $D$, Bitterlich N, Domhardt R, Foth D, Grässler A, Michalak U, Schicke B, SchmidtPich J, Schulz J, Zimmermann T (2003) Die Gesundheit der Frau: Klimakterium, Vitalität und Hormonsubstitution - Ergebnisse einer funktionsdiagnostischen Pilotstudie.J Menopause 10(3):20-27

Hinweis des Verlags. Der Verlag bleibt in Hinblick auf geografische Zuordnungen und Gebietsbezeichnungen in veröffentlichten Karten und Institutsadressen neutral.

\section{Zusammenhang zwischen Brustkrebs und Knochen- wachstum}

Knochen wachsen als Reaktion auf bestimmte Signalmoleküle von Brusttumoren: Dies hat ein Forscherteam des Max-Planck-Instituts für Kolloid- und Grenzflächenforschung (MPIKG) und Biologen der Cornell University, USA, herausgefunden und damit einen möglichen Abwehrmechanismus gegen Knochenmetastasen entdeckt.

Welche Mechanismen die Entstehung von Knochenmetastasen fördern, ist bislang wenig erforscht. Bekannt ist, dass Tumorzellen verschiedene Signalmoleküle aussenden und somit Organe für die Ausbreitung von Krebs anfällig machen. Bekannt ist auch, dass Brustkrebs mit Knochenmetastasen Knochenabbau verursacht, da metastatische Tumoren knochenabbauende Zellen aktivieren und knochenbildende Zellen hemmen.

Unter Leitung von Prof. Dr. Peter Fratzl machten die Potsdamer Materialwissenschaftler des MPIKG nun eine überraschend gegenteilige Entdeckung: Untersuchungen an einem von der Cornell University entwickelten Tiermodell zeigten, dass in einem frühen Stadium Knochen schneller wachsen, wenn sie Signalmolekülen des Tumors ausgesetzt sind. Dies ist möglicherweise ein Abwehrmechanismus des Körpers, der eine Metastasenbildung zu verhindern versucht. Die Studienergebnisse könnten zukünftig diagnostische Tests und therapeutische Brustkrebsbehandlungen beeinflussen.

Das interdisziplinäre und internationale Forschungsprojekt wurde vom „Human Frontier Science Program" mit 1 Million Dollar gefördert.

Quelle: Max-Planck-Institut für Kolloid- und Grenzflächenforschung (MPIKG), https://www.mpikg.mpg.de/ 
Hier steht eine Anzeige.

\section{曾 Springer}

